\title{
sciendo
}

Transport and Telecommunication, 2019, volume 20, no. 4, 365-378

Transport and Telecommunication Institute, Lomonosova 1, Riga, LV-1019, Latvia

DOI 10.2478/ttj-2019-0030

\section{BLOCKCHAIN AND SMART CONTRACTS FOR ENTREPRENEURIAL COLLABORATION IN MARITIME SUPPLY CHAINS}

\author{
Robert Philipp ${ }^{1,2}$, Gunnar Prause ${ }^{1,2}$, Laima Gerlitz ${ }^{2}$ \\ ${ }^{I}$ TALTECH University \\ Ehitajate tee 5, 12616 Tallinn, Estonia \\ ${ }^{2}$ Hochschule Wismar, University of Applied Sciences: Technology, Business and Design \\ Philipp-Müller-Str. 14, 23966 Wismar, Germany \\ robert.philipp@hs-wismar.de \\ gunnar.prause@taltech.ee \\ laima.gerlitz@hs-wismar.de
}

\begin{abstract}
Smart contracts are scripts on the top of the blockchain technology. They represent a form of automation by what the layers of intermediaries can be reduced or even completely replaced. Accordingly, blockchain smart contracting systems decrease transaction and enforcement costs as well as process time.

Moreover, we argue, blockchain and smart contracts can facilitate cross-organisational collaboration and their underlying business processes. Hence, they are able to support the integration of entrepreneurs and SMEs into trans-national supply chains by reducing high entry barriers and weakening the dominating position of big players.

This paper discusses the research questions how blockchain smart contracting can facilitate the implementation of collaborative logistics structures and how the integration of SMEs into sustainable maritime supply chains can be safeguarded. The research bases on expert interviews and case studies. The results showcase the potentials of using blockchain smart contracting in the environment of trans-national and multimodal supply chains.
\end{abstract}

Keywords: Blockchain, Smart Contracts, Maitime Supply Chains, SMEs, Ports, Entrepreneurial Collaboration

\section{Introduction}

Supply chain management targets to integrate different organisational entities of a supply chain as well as to manage efficiently materials, information and financial flows for greatly fulfilling customer demands with the objective of enhancing competiveness of the entire supply chain (Stadtler, 2005). Thereby, supply chains among other things refer to the interactions with suppliers, warehouses, distribution centres, retail outlets, raw materials, goods-in-process stocks and finished products (SimchiLevi et al., 2003). Accordingly, due to many different interest groups, sophisticated business processes as well as distributed structure of supply chains, the management of the supply chain flows remains a challenging task (Prause, 2019). Moreover, further "evergreen" challenges in this context are related to the dominating position of big players, establishment of trust and relationships between the different actors, fragmented structures, supply chain finance and lead-time as well as throughput (Beth et al., 2013; Nyhuis and Wiendahl, 2008; Prause and Hunke, 2014; Zhao and Huchzermeier, 2018). In order to smartly tackle these issues, the current research efforts in logistics result into the evolvement of potential reorganisations for an efficient crossover from scattered supply chains to open logistics networks, where resources are accessible, compatible and intertwined (Rusich, 2018). Thus, in the context of Industry 4.0, a shift towards smart manufacturing with a focus on fractals, networked cyber-physical systems (CPS), self-organisation, self-optimisation and machine-to-machine systems (M2M) is noticeable (Prause and Atari, 2017). Therefore, challenges in the course of supply chain management become smarter, more networked, fragmented, decentralised and distributed (Prause, 2015; Olaniyi and Reidolf, 2015). This transition culminates in the new paradigm Logistics 4.0, also well-known as smart logistics, which can be understood as a management approach for developing, designing, managing and realising change-oriented networks of object flows (e.g. materials, information, values) based on pattern recognition, generalisation and self-organisation, enabled through the usage of new technologies and innovative services (Wehberg, 2016). In this context, among all novel technologies, one of the most promising approaches with far reaching potentials is the blockchain technology including smart contracts. 
Smart contracts are computer codes that run on the top of the blockchain technology. They are a very simple form of automation with "if-then-else" functions. Accordingly, smart contracts represent transactional protocols or scripts that can execute and enforce legal contracts. Thereby, they selfexecutable permanently audit the achievement of pre-defined and previously agreed terms and conditions that are coded and stored on the blockchain ledger, including new uploaded and up-dated data and documents. Through this automated reconciliation, smart contracts can trigger in real-time respective actions or transactions, respectively, as soon as the pre-defined contractual clauses and rules are fulfilled.

In the course of supply chain management high interest is given, since especially information intermediaries can be reduced or fully replaced by the implementation of blockchain smart contracting systems. Furthermore, smart contracts and in particular, the underlying blockchain technology, bear the potential to decrease transaction and enforcement costs along supply chains. Building upon this, we further argue that smart contracts applications on a blockchain platform in the areas of logistics networks and supply chains support the integration of entrepreneurs and SMEs into trans-national value and supply chains, which are currently still closed due to high entry barriers or to domination of big players (Prause and Hunke, 2014; Prause and Hoffmann, 2017).

The authors took place in several EU-projects with focus on green logistics and sustainable supply chain management, and where resulting research studies confirmed that especially in the context of Industry 4.0, SMEs can benefit (e.g. Prause and Atari, 2017; Gerlitz, 2017, 2015). Hence, the current research paper discusses the research question how smart contracting and blockchain technology can facilitate the implementation of collaborative logistics structures and how the integration of SME sector into sustainable trans-national maritime supply chains can be safeguarded. To address this issue, we use two case studies. The first case study refers to a simplified charter-party contracting process within a single voyager in the freight market, whereas the arising advantages of an adequate technology implementation are highlighted. In contrast to this macro-logistics level perspective (i.e. port environment), the second case study concentrates on a micro-logistics level, whereby the immediate port sector - cargo import case - is addresses. Hence, the case study of the medium-sized seaport of Wismar is used to showcase the potentials of blockchain technology and smart contracts to simplify the port logistics processes with increased efficiency.

Generally, the research study refers to expert interviews and case studies from several EU-projects with a focus on the two ongoing projects "Connect2SmallPorts" and "CSHIPP", which are implemented in INTERREG VA South Baltic and INTERREG VB Baltic Sea Region programmes. The research findings will highlight that smart contracts and blockchain technology create great advantages for SMEs, since their competiveness and efficiency can be enhanced and participation ensured through the adoption of this new technology due to lower transaction costs and disintermediation.

The paper is structured as follow: The second chapter provides insights to the blockchain and smart contract technology by a literature review. Building upon this, the research method is set out. In the fourth section, the research findings and discussion based on the two case studies of a (1) charter-party contracting process within a single voyager in the freight market, and (2) the medium-sized seaport of Wismar are showcased. The paper rounds up with a conclusion.

\section{Literature Review}

Intellectual roots of the blockchain technology can be traced back to Satoshi Nakamoto in 2008 (Nakamoto, 2008). Blockchains are comparable with open and distributed ledger systems that facilitate via alphanumerical code (i.e. address account) and private key the secured execution of transactions in a virtual network with untrusted parties. Thereby, for the cryptocurrency exchange intermediaries are superfluous, which represents one of the special features of the blockchain technology that among other things ensures the development of a decentralised network of trust (Chuen, 2015; Gallay et al., 2017; Liao and Wang, 2018; Manski, 2016; Swan, 2015). This is, because each independent participant receive a copy of the ledger for own control purposes. Accordingly, next to intermediaries, this distributed virtual ledger system can supplant physical documents as well as signatures. As highlighted by Wu (2018), especially this decentralised and distributed feature of the blockchain technology can foster the competiveness and efficiency of entrepreneurs and SMEs through enhanced information accessibility, reduced risks and levels of intermediaries, which result in a decrease of transaction and intermediary costs. Accordingly, by taking one-step further, it can be argued that the blockchain technology bears the potential to facilitate entrepreneurial collaborations in trans-national supply chains.

Smart Contracts - also known as digital contracts or e-contracts - expand the initial blockchain application and suggest wider use cases (Gallay et al., 2017; L'Hermitte et al., 2018; Swan, 2015; Wu, 2018). 
They represent transactional scripts or protocols that include terms and conditions of contracts. Building upon on a blockchain platform, where they are implemented through digital codes with software, smart contracts automatically process the negotiated conditions of contracts to which all involved parties previously agreed (Liao and Wang, 2018). Accordingly, they are stand-alone programmes that audit the pre-defined conditions of an agreement, which was concluded between at least two parties. Furthermore, they are able to conduct reconciliation with new transactions - or even recent uploaded and updated data and documents that had been added to the blockchain ledger by IoT applications. This procedure is permanently self-executable performed by smart contracts. As soon as all pre-defined terms and conditions are fulfilled, smart contracts automatically trigger a certain number of actions or transactions dependent from the purpose they were originally programmed (i.e. according to their script) (Kouhizadeh and Sarkis, 2018).

With other words, smart contracts are able to read from and write on the blockchain. In addition, they ensure that whenever a certain transaction or action occurs, further transactions or actions are conducted automatically (García-Bañuelos et al., 2017). Therefore, it can be stated that smart contracts ease and validate the completion of contractual agreements. Thereby, they represent a very simple form of decentral automation with "if-then-else" functions. As a result, the intermediaries and the existence of trust in the area of contractual agreements become superfluous (Liao and Wang, 2018; Kouhizadeh and Sarkis, 2018). All this fosters the development of DOAs (decentralised autonomous organisations) (Manski, 2016).

To summarise, the contractual conditions as well as related legal principles are coded as algorithms in smart contracts, which are transparent, since they are stored on the decentralised and distributed blockchain network, and thus, shared with their digital record and signature under the contract parties (Kouhizadeh and Sarkis, 2018). Through this, the characteristics of the blockchain technology effect smart contracts and make them more protected against distortions, revisions and manipulations as well as deletion (Liao and Wang, 2018). Since also the utilisation of smart contracts further incorporates the potential to remove the value of interposed or so far necessary intermediaries (e.g. third parties, middlemen, brokers or agents like governments, banks, lawyers, etc.) in various business activities, they can additionally decrease transaction costs as well as facilitate the efficiency and redesign of complex business structures that are characteristic for supply chains.

\section{Method}

The research on smart contracts and blockchain technology in the nexus of maritime logistics sector, and in particular in the context of small and medium-sized seaports, have not sufficiently investigated so far (Liao and Wang, 2018). Hence, to the best of our knowledge, currently there exist no published work that discussed how smart contracting and blockchain technology can facilitate the implementation of collaborative business structures for sustainable trans-national entrepreneurial activities in maritime supply chains. According to Creswell and Creswell (2017), if a concept or phenomenon needs to be understood, because less research has been done on a specific topic, especially the qualitative research approach is suitable. Therefore, in the frame of the current research study, the decision was made to favour a qualitative research approach. Nevertheless, the research was performed based on simultaneous procedures, where quantitative as well as qualitative data was synthetized in order to efficiently tackle the identified research problem (ibid.). Hence, the authors gathered primarily qualitative as well as quantitative data, and combined the received information in the frame of the interpretation process that is reflected by the presented holistic research results. The qualitative research in the present research study represents a narrative research. In the course of narrative research, the gathered information from experienced individuals is condensed and retold by the researchers (ibid.). The authors of the present research study performed 21 semi-qualitative expert interviews with relevant project experts, scientists as well as project interest groups that took place during November 2018 and March 2019. These expert interviews mainly embraced open-ended questions, whereby a quarter of the questions represented closed-ended questions that grounded on a five-point metric scale. Building upon this, two case studies were performed in the current research study. The research was complemented by field research and observations, an extensive literature review, analysis and examination of respective theories and approaches, topic-related policy regulations and guidelines, representing a systematic research procedure for the elaboration of a process, action and/or interaction about a substantial subject (ibid.). By doing so, the researchers identified common functionalities of a sustainable usage of blockchain smart contracting systems (1) in the context of a charter-party contracting process within a single voyager in the freight market, as well as (2) in the immediate environment of a medium sized 
seaport; and thus, were able to present the advantages of the technology adoption as well as related grassroots guidelines for an efficient implementation.

In frame of case studies, programmes, events, activities, processes or individuals are investigated in depth (Creswell and Creswell, 2017; Yin, 2009), where profound information is gathered by using a wide range of data collection activities in a certain time frame (Stake, 1995). In the course of the presented case studies in this research paper, the researchers got involved with the primary target group that is defined by: policy makers that are responsible for infrastructure and port development; port and terminal operators; forwarders; international organisations and institutions that refer to port-related supply and value chains; shipping companies and/or lines; research institutions; local and regional industries that are interested in digitalisation issues and governmental investments as well as higher data security.

The focus of the empiric research activities laid on the following ongoing INTERREG projects: (1) "Connect2SmallPorts" and (2) "CSHIPP". The Connect2SmallPorts project focusses on improving cross-border connectivity for a functional blue and green transport area, with the objective to improve the quality and environmental sustainability of transport services in South Baltic Sea Region. In contrast, the CSHIPP project focalizes on sustainable transport, with the objective to enhance clean shipping based on increased capacity of maritime actors of the whole Baltic Sea Region (i.e. environmentally friendly shipping). Through active collaboration and in some cases leadership (Connect2SmallPorts) by the researchers in the different cross-border research projects, access to a wide range of data and insights on the research topic is ensured, which was crucial and the stepping-stone for the current research. Next to the public stakeholders, especially, the private organisations that participate as project partners in the EUfunded projects delivered essential insight into their business practises and the status-quo of blockchain and smart contracts in the environment of maritime logistics sector and specifically in the context of small and medium-sized ports.

Relevant information and observations were gained from a broad field of project actions like project partner meetings, workshops, trainings, matchmaking events as well as logistics and open seaportrelated conferences with project interest groups. In addition, focus group meetings had been performed for a stronger target-oriented investigation of particular topics of interest. These focus group meetings were mainly conducted in the course of the EU-project Connect2SmallPorts, which also targets to explore use cases and new business models that arise from the potential application of the blockchain technology in the context of small and medium-sized seaports in South Baltic Sea Region. Moreover, additional primary information were gathered from individual meetings with experts from the logistics and IT sector, which supported the collection of information about the presented cases, related challenges and potential implementation procedures. These meetings were conducted with decision makers of the ports from Wismar (DE), Rostock, Stralsund, Vierow, Karlskrona (SE) and Klaipeda (LT). At the same time, phone conferences and Skype meetings assisted this data and information collection process. The logistics and seaport-related conferences, where the authors participated, spurred the information exchange with further stakeholders. Hence, the exchange and collaboration with numerous different stakeholder groups allowed the achievement of a more comprehensive perspective on the topic.

To sum up, the qualitative research approach safeguarded the examination of the identified research problem in a comprising manner. The novel topic - blockchain smart contracting system - was described fundamentally by the literature review. Building upon this, the potential adoptions of this new technology in the context of (1) charter-party contracting processes, as well as (2) small and mediumsized seaports is described in the following in form of use cases. Thereby, observations, expert interviews, focus group meetings and case studies were used as the main methods of the present research study. Based on the presented research findings, the authors were capable to achieve the indicated research objective and to answer the related research question in a qualitative manner. Lastly, the presented research results were validated and verified by the abovementioned primary target groups in the course of project-induced workshops, trainings and seminars.

\section{Findings and discussion}

The subsequent first case study exemplifies a simplified charter-party contracting process within a single voyager in the freight market, whereby the arising advantages of an appropriate technology implementation are emphasized. Contrary to this macro-logistics level perspective (i.e. port environment), the second case study focus on the micro-logistics level, since the immediate port sector - cargo import case - is described. This illustrated cargo import case is characteristic for the medium-sized seaport of Wismar in Germany, but to a certain degree also applicable or generalizable, respectively, to other seaports that focus on break-bulk cargo. Through this, a clear presentation of emerging potentials that are 
achievable through the adequate implementation of the blockchain technology and smart contracts is ensured and result in simplified port logistics processes with increased efficiency.

\subsection{Charter-party contracts}

In order to exemplify the various benefits that arise from a potential implementation of the blockchain technology and smart contracts on a macro-logistics level, we use and describe a simplified charter-party contracting process within a single voyager in the break-bulk market. In a first step, the present situation in the freight market is presented, which is followed by an in-depth analysis and exposition of achievable improvements in order to arrive at an optimised process flow, which can be ensured by the integration of a potential blockchain smart contracting system. The general charter-party contracting process comprises four phases: (1) pre-fixture, (2) fixture, (3) post-fixture loading and (4) post-fixture discharging.

In the pre-fixture phase, the process generally starts with a charterer, who has a fixed sales contract with a supplier or processors - for e.g. wood logs (i.e. break-bulk cargo). Since in the charter-party contracting process many different actors are involved, in our simplified case the charterer is also the trader and/or shipper, or at least closely related to them. In order to deliver the cargo, the charterer seeks for a vessel that is suitable to transport the respective freight. Accordingly, the charterer wants to conclude a contract with a ship-owner. The task to find a suitable ship for a specific type of cargo is usually delegated to a shipbroker, whereby generally both parties (i.e. charterer and ship-owner) have their shipbrokers, and even the interposition of a third shipbroker between the charterers' and shipowners' shipbrokers is also possible. However, usually the charterer appoints the shipbroker, who checks the current market situation. In order to perform this task, the charterer has to provide the shipbroker with initial information regarding name and address as well as contact details of charterer, cargo type and quantity as well as physical dimensions, loading and discharging ports and rates, expected laycan, etc.

Subsequently, in the fixture phase, the shipbroker seeks for a suitable ship according to the indicated freight features, desired period of time as well as expected rate(s). Accordingly, the shipbroker contacts several ship-owners and starts the negotiation process on behalf of the charterer. Depending on the specific conditions of the envisaged contract, the individual market situation and the network of the shipbroker, this search process may take some hours, but often a few days or even weeks. Finally, when the shipbroker found a suitable vessel according to the desired conditions, the offer with initial terms and conditions from the charterer is send to the ship-owner by the shipbroker. Then, the ship-owner checks the received offer and conducts respective pre-calculations, which result in the sending of the initial time charter or freight rate (here simplified as the general charter rate) to the shipbroker, who then forwards it to the charterer, whereby in some cases the appointed shipbroker also has an initial charter rate idea. The process of negotiation starts and can result in a consensual agreement, a counteroffer, or a disagreement. Accordingly, this procedure may takes some time due to potential emergence of circulating counteroffers, whereby the additional problem occurs that the related documents and/or offers are send back and forth - often still via a courier. Generally, the shipbroker function as the information intermediary between the charterer and the shipowner. Hence, each decision or information of the charterer or ship-owner is firstly shared and communicated with the interposed shipbroker, before it is forwarded to the charterer or ship-owner. When the charterer and ship-owner finally agreed to the negotiated charter rate, terms and conditions, the shipbroker drafts the respective contract that includes the aforementioned information, which is then forwarded to the charterer and ship-owner for final fixture. Accordingly, the charterer and ship-owner audit the received charter-party contract form and if all necessary information are correct and included, they sign the contract. Usually sound charter-party contracts are quite comprehensive, since they cover many important and crucial information, e.g. details regarding the ship-owner and charterer as well as shipbroker, ship details, brokerage fee (commission), charter rate, cargo and carriage specifications, loading and discharging ports and rates as well as dates, laytime, demurrage and despatch rates, freight payment, payment terms and details including currency, and other obligations and rules as well as clauses. Hence, charter-party contracts also contain legal aspects and clarify responsibilities, also for cases of unexpected events and rules that apply in case of non-performance. Due to the complexity and wide range of potential uncertainties that must be hedged in charter-party contracts, in practice, there exist typical standard templates. For instance, in case of voyage charters common standard charter-party contract forms that are used are among other things NUBALTWOOD, GENCON, etc. Vice versa, in case of time charters wellknown standard forms are ASBATIME, BALTIME, GENTIME, LINERTIME, NYPE, etc. These templates cover the most relevant clauses that must be taken into account when concluding a charter-party contract.

In the post-fixture loading phase primarily monitoring tasks are performed. Usually, both the charterer and the ship-owner are not personally present at the loading as well as discharging ports. Hence, 
port agents are appointed at the exporting and importing ports, who are equipped with the necessary information, instructions and rights. Related decisions are also negotiated and then incorporated in charter-party contracts during the fixture phase. Accordingly, the port agent at the origin port monitors the lading sub-process, stevedores, bills of lading, notice of readiness statement (NOR) and vessel's statement of facts (SOF), etc. In addition, he sends progress reports about the status to the charterer and ship-owner, as well as checks and transmits the freight receipt.

The post-fixture discharging phase on a first glance similarly embraces mainly monitoring tasks. Accordingly, in the frame of the discharging sub-process at the destination port, the port agent controls the discharging progress, notice of readiness statement (NOR) and vessel's statement of facts (SOF), payment status, etc. This includes as well the transmit of progress information to the charterer and shipowner. When the vessel is discharged, the charterer and ship-owner start to conduct concluding calculations according to the previously agreed terms and conditions in the charter-party contract and actual performance. If the charterer and the ship-owner come to a joint and concluding outcome, the charterer pays the ship-owner and the ship-owner arranges the payment of the shipbroker, regardless whether the charterer appointed the shipbroker and independently whether more than one shipbroker was involved in the process. Vice versa, if the ship-owner and the charterer do not reach a joint consensus in the frame of the performed concluding calculations and thus, the respective receipt, a dispute may arise, which can result in additional delays regarding the payment sub-process. This can lead to the involvement of the shipbroker as a mediator or lawyers and courts. Therefore, in order to avoid such problems, it is essential that the previously concluded charter-party contract is detailed and robust.

The described general charter-party contracting process incorporates many options for enhancements that can be partly empowered through the implementation of the blockchain or smart contract technology, respectively. Hence, for enhancing the charter-party contracting process, a blockchain driven smart contracting system is proposed, which targets among other things to directly connect the charterers and the ship-owners. Next to this, especially the information and cargo flow of the overall supply chain, as well as entrepreneurial collaborations can be improved.

In the course of the pre-fixture phase, the charterer is looking for a suitable vessel and the shipowner searches for freight that needs to be transported from an origin to a destination port. Thereby, relevant information from charterers' side are cargo type and quantity as well as physical dimensions, loading and discharging ports, expected laycan, etc. Against this, the well-needed information from shipowners' side are ship type and size, current position, period of availability, etc. In the abovementioned current situation, usually the charterer appoints a shipbroker for the generation of offers according to the respective situation on the market - in our case the respective situation on the break-bulk market. Therefore, the shipbroker administers the received information of both parties, and based on this, seeks for suitable matches. This initiation phase can be eased by an information-sharing platform that is implemented via the internet. Such comparable platforms already exist, whereby one of the most prominent representatives is OpenSea.Pro, which is a web-based chartering marketplace system that simplifies the tasks of shipbrokers. This is, because the platform eases the monitoring of the respective situation on the global freight market in real time, streamlines the search process for matches through the entered and stored data of ship-owners and charterers, as well as facilitates the communication process with both parties and the shipbroker. Accordingly, in practice, there exist already virtual marketplaces, where ship-owners and charterers could easily find each other and make contact, if their uploaded profile, entered data, conditions and requirements are of interest for at least one party. Thus, these platforms are able to connect the ship-owners and charterers directly without the necessity to interpose a shipbroker. Especially this can streamline the matchmaking sub-process, which shortened time, eases the communication as well as ensures the exchange of data and information - even in later stages of the cargo voyage. Moreover, this pre-fixture phase that can be facilitated through the implementation of a virtual marketplace also represents the potential initial starting point for the realisation of a blockchain and smart contract application. For instance, the virtual marketplace can be implemented and work based on the blockchain technology with additional smart contract applications. A smart contract application could compare the shared initial data from charterers and ship-owners that is provided on the web-based marketplace platform, and thus, can automatically check whether the pre-conditions for a match are fulfilled. Building upon this, a kind of pre-contracts can be automatically generated as well by the smart contract application, since the shared data of the participants represents at the same time the initial information and pre-conditions that must be fulfilled for the aspired development of a charter-party contract.

The fixture phase is primarily characterised by negotiation activities between the ship-owner and the charterer, whereby the overall information flow is organised via the interposed shipbroker. Once a 
suitable ship was found according to the pre-defined conditions, the shipbroker sends the initial offer to the ship-owner. Then, the ship-owner audits the received offer and conducts respective pre-calculations, which can result into the sending of the calculated charter rate to the shipbroker, who forwards it to the charterer. Generally, this negotiation process can result in a consensual agreement, a counteroffer, or a disagreement, and thus, can be extremely time-consuming, as this process is not automated. However, when the ship-owner and charterer agreed to the charter rate and other terms and conditions, the contract will be prepared by the shipbroker according to the previous negotiated and agreed rates and clauses for final fixture. Also in this process phase, the implementation of a blockchain smart contracting system would achieve great advantages. For example, Norta (2015) developed a smart-contracting setup lifecycle for the negotiation procedure, which can be seen as a viable foundation for the transition to a smart charter-party contracting system. On the other hand, the wide acceptance in practice and thus, usage of standard charter-party contract forms additionally encourages the potential of automation via smart contracts, since both actors (i.e. ship-owner and charterer) appreciate that such templates for charter-party contracts cover the most important clauses that need to be taken into account. Furthermore, indices like the Baltic Dry Index that are used in practice function as orientation (e.g. hurdle rate) in the course of price negotiations. This respective market data as input data can be automatically integrated in the smart contracting negotiation process. Once the charterer and the ship-owner have jointly agreed on the rates and terms, the charter-party agreement is fixed, and is automatically generated through the underlying smart contract application. Moreover, the elaborated and fixed charter-party contract is stored and secured on the blockchain. Accordingly, the fixed charter-party contract becomes decentralised secured, fraudresistant, immutable, transparent and permanently auditable and accessible for all involved stakeholders during the cargo voyage - if permission is granted.

In the post-fixture loading phase, primarily monitoring activities are performed that are mainly in the area of responsibility of the appointed port agent at the loading port. These monitoring tasks are related to the loading progress, stevedores, bills of lading, notice of readiness statement (NOR) and vessel's statement of facts (SOF), etc. The status information about the port operations are forwarded to the ship-owner and charterer by the port agent, and function as important input data for the concluding calculations in the post-fixture discharging phase. In the actual situation, this embraces the back and forth sending of physical documents, copies and time sheets between all involved parties. Accordingly, also in this phase, the potential integration of the blockchain technology can optimise the process flow. For instance, by the additional incorporation of IoT applications - especially smart devices - the respective information about the loading activities in the origin port can be uploaded and stored on the blockchain in real-time. Through this, all necessary information or documents, respectively, become decentralised secured, fraud-resistant, immutable, transparent and permanently auditable and accessible for all involved parties. Since smart contracts can read from and write on the blockchain, important documents like vessel's statement of facts (SOF) or bills of lading can be automatically elaborated by an implemented smart contract application - if all pre-defined conditions are fulfilled and thus, all relevant data and information is available on the blockchain ledger through further uploaded, secured and shared files.

During the post-fixture discharging phase, comparable monitoring tasks occur as in the postfixture loading phase, which result again in the flow of information between all involved participants. Therefore, also in this sub-process, blockchain smart contract applications are reasonable: IoT and smart contract applications feed the blockchain ledger with needed input data and smart contracts process these information and data in order to trigger further transactions or actions, respectively. Furthermore, next to the monitoring activities, this phase includes the concluding calculations, which need to be performed by the ship-owner and charterer. This is conducted based on the formerly fixed charter-party contract as well as all collected information and data that was generated during the entire voyage of the freight. Generally, this should result in the payment of the ship-owner for his service. These activities can be optimised, since they can be automated via an implemented blockchain smart contracting system. In addition, the calculation process of all related expenses like demurrage and despatch can be automated via smart contracts, too, since the needed rates are recorded in the charter-party contract, which is secured on the blockchain ledger in our optimised process. Moreover, all other important input data that is needed for the concluding calculations via implemented smart contract application, is provided in our optimised process flow through the incorporated IoT applications. Accordingly, building upon all secured and thus, available information and data on the blockchain ledger, which was generated by all involved process parties during the entire cargo voyage as well as IoT applications, a smart contract application is able to elaborate automatically also the respective invoices - next to the automated calculation of related expenses. Additionally, if all conditions and requirements are fulfilled, the smart contract application is also able to trigger automatically the related transactions (i.e. payments) in accordance to the 
automatically generated invoices. Ensured through the implementation of a smart contracting system in the concluding calculation and payment process, the emergence of objections by the ship-owner and charterer can be limited, due to the decentralised nature of the blockchain and smart contract technology. Additionally, this is also deeply rooted in the fact that the automated expense calculations lie on the documents and data that had been worked out by the port agents, as well as on the formerly agreed and sign charter-party contract, which includes all agreed rates and terms. Accordingly, this procedure guarantees a trustful and fair approach for automated concluding calculations. Hence, a dispute between the ship-owner and charterer is less likely. Vice versa, this makes the involvement of courts and lawyers, or the shipbroker as a mediator superfluous, which at the same time prevents against additional delays of the payment sub-process and thus, the overall process.

The benefits of an implemented blockchain smart contracting system in the ecosystem of a virtual marketplace are manifold. Through the incorporation of IoT applications, the cargo flow becomes trackable and the needed data generation for the smart contract applications is ensured, whereby among other things the integration of GPS shipping data could even foster this development. Moreover, the data exchange between incompatible systems is avoided and the data transfer becomes digital and automated, which result in a higher flexibility, streamline of the process and saves time, since no back and forth sending of physical documents via courier is necessary. Hence, all relevant documents and data are stored on the blockchain ledger, which guarantees availability at all times and for all involved stakeholders during the entire cargo voyage. Accordingly, this replaces the physical paperwork (i.e. multiple versions of files, signatures, etc.), whereby each party can digitally sign via a private key, validate files and generate copies, if necessary. Since during each cargo voyage, many but every time different actors are involved, a permissioned blockchain would be an appropriate solution. This is reasoned by the fact that within a permissioned blockchain, it is possible to clarify access and modification rights of the participants as well as proof of personal identification. Accordingly, specific transactions or actions can be set as private to certain participants. For instance, this might be useful in case of price negotiations and the result thereof. Generally, this can be regarded as a data filter, which ensures that only certain authorised parties are allowed to add, receive or inspect respective data and information that is needed for further tasks in their area of responsibility. Moreover, a simultaneous document processing and process tracking can be achieved, which supports the overall process transparency. Besides this, through the blockchain storage, all relevant documents become decentralised secured, fraud-resistant, immutable, transparent and permanently auditable and historically retraceable as well as accessible for all involved and authorised parties during the cargo voyage. This fosters trust among all involved actors and enhances efficiency of the entire process due to a higher flexibility. In addition, this blockchain recording procedure, delivers great fields of application for smart contract programmes. For instance, important documents such as the charter-party contracts can be generated automatically, if all pre-defined conditions are fulfilled that are available through the shared and stored data on the blockchain ledger. This includes as well the automation of the auditing procedures in case of documents and negotiations, as well as the inclusion of external available market data - e.g. the Baltic Dry Index on the freight market for price negotiations. Lastly, concluding calculations can be also automated by a smart contract implementation, as the necessary data basis is available through the underlying blockchain ledger. Through this, a smart contract application is able to automatically elaborate the final invoices, and if all conditions are fulfilled can trigger automatically the related payments. Hence, a blockchain and smart contract installation ensures a trustful and fair approach for the calculation of expenses as well as for monetary transactions. To sum up, all these highlighted aspects decrease costs due to shortened process time and lower manual works.

However, next to all showcased benefits, in particular the potential exclusion of the shipbroker as the central information intermediary between the ship-owner and charterer can have far-reaching positive impacts on maritime supply chains. It is obvious that through the introduction of a blockchain smart contracting system for the generation and conduction of charter-party contracts in the ecosystem of a virtual marketplace, the shipbroker becomes superfluous. With other words, the implementation of a smart charter-party contract system replaces the shipbroker and thus, reduces the layers of intermediaries in maritime supply chains, since the direct connection of the charterer and ship-owner is ensured. Enabled by the smart contract automation on a web-based marketplace, the ship-owners can transparently show their service supply and period of availability to the charterers, who can similarly indicate their timebound service demand to the ship-owners. All this can be achieved without the necessity of an interposed shipbroker. Through this, the information flow is shortened, which in addition saves time. The formally given trust through the presence of the shipbroker is compensated by the decentralised nature and further benefits of the introduced blockchain and smart contract technology, which automatically leads to the 
emergence of trust among all involved users. Moreover, through the exclusion of the shipbroker, there are cost savings that have potential spill-over effects. For instance, as a direct impact, the brokerage fee or commission is saved by the ship-owner. Depending whether a voyage charter or a time charter is achieved, the involved shipbroker receives a commission on the gross freight or hire price for the vessel. Next to this, the brokerage fee is also dependent form further factors, such as type of cargo, kind of fixed ship, negotiations and agreements with the ship-owner, etc. Nevertheless, within voyage charters the commission usually varies between 1 and 5\%. Contrary to this, within time charters, the brokerage fee amounts typically $2.5 \%$ of the hire price. Since the ship-owner has to pay the shipbroker, this causes direct cost savings for the ship-owner. As a side or spill-over effect, the ship-owner might be able to lower the charter rate, which would be beneficial for the charterer. On the other hand, this could also result in higher payable loading and discharging rates at the ports. Therefore, the absence of the brokerage fee could also indirectly have positive effects to all involved actors - e.g. also for seaports through higher wharfage and port dues. Accordingly, the exclusion of the shipbroker is adding value for all stakeholders along the maritime supply chain. In particular for smaller and entrepreneurial actors, this enhances competiveness, improves efficiency and enables participation through the enabling adopting of this novel technology due to potentially emerging spill-over effects.

\subsection{Cargo import in ports}

In the maritime logistics system, three main actors can be pinpointed: (1) shipping companies, (2) ports and (3) freight forwarders (Caliskan and Ozturkoglu, 2018). Hence, the global movement of freight is not realizable without the complementary actors to merchant shipping known as seaports. Seaports are the main hubs for commercial operations worldwide, and thus, each port offers a value proposition to its surrounding region since they provide economic and social benefits (Rodrigue and Schulman, 2013). In fact, seaports are the backbone of the transport network without todays' worldwide economy could not exist in its present form (Funke and $\mathrm{Yu}, 2011$ ). If all economic activities that depend on the sea would be cumulated, the so-called "blue economy" of the EU is responsible for about 5.4 million jobs and a gross value added of almost 500 billion EUR per year (European Commission, 2017). 74\% of goods imported and exported, and $37 \%$ of exchanges within the EU transit through seaports (Pastori, 2015). In 2017, almost four billion tonnes of cargo and 415 million passengers passed through the 1,200 European seaports (Eurostat, 2019a, b).

Accordingly, in order to manage the huge freight and passenger demand, the necessity to enhance efficiency in the shipping and port industry increases steady. Especially for port logistics, a safe and reliable data flow is crucial for efficient processes - in particular, when different cargo types shift between distinct transport modes. In order to achieve a smooth operational process flow, real-time data and open data access for all involved parties is essential. Nevertheless, in practice, many port processes are still quite old fashion, which for instance is mirrored by information exchange via telephone or physical paper documentation and forwarding. This is especially true for small and medium-sized seaports that have a common and high interest in digitalisation issues, but among other things are regularly not able to secure financing for their sustainable and competitive development and that are often neglected by scientists and policy makers on EU, national, regional or even local level (Philipp et al., 2018; Rozmarynowska and Oldakowski, 2013).

The usage and implementation of blockchain technology in the frame of port logistics bears the potential to facilitate the transition from a paper documented process management to a digitalised and more secured one by validating and storing each action or transaction, respectively, in the chain of blocks. Accordingly, the optimisation of the financial, cargo and information flow within port logistics through blockchain and smart contract applications exhibit great potentials, even in small ports with less financial resources. To showcase the advantages that arise from a blockchain and smart contract usage in the immediate port sector, the possible implementation of these novel technologies has to be demonstrated and compared with the current situation. Therefore, building upon the findings from the EU-project Connect2SmallPorts, in the following, the case study of the seaport of Wismar in Germany is used as an example.

Wismar seaport in the sense of the analogy of the TEN-T is a comprehensive port and can be classified according to its characteristics as a medium-sized seaport. The geographical position of Wismar seaport fosters mainly the north-south traffic of cargo between Central Europe and Scandinavia. Nevertheless, the site factor also leads to the primary focus of collection and distribution of cargo flows that can be traced back to the east-west connection to the Baltic States and Russia. Wismar seaport is specialised in handling and storing bulk and break bulk cargoes like wood logs and forest products, metals and scrap, building material, salt and fertilizers, as well as project cargo. Its port logistics for 
energy sources like wood pellets and wood chips represents an important link in the biomass value chain, whereby the seaport is active in both import and export of the related raw material, which at the same time classifies his leading role in the Baltic Sea. Hence, Wismar seaport is an important partner of many sawmills across Europe. However, especially the local timber cluster in the near proximity of the port and in the adjusted hinterland plays an important role for the seaport; and vice versa the port for the local and regional industry and economy. Since for the seaport and the local timber cluster, the import of raw materials - here in particular wood $\operatorname{logs}$ - from Scandinavia has a high relevance, in the following, the cargo import case is analysed more detailed. As indirectly and abovementioned in the previous case study, the cargo import case involves many different actors comprising intermediaries like port agents and shipbrokers as well as freight forwarders; ship-pilots; tug masters; inshore pilots; port office and/or authority; terminal/port operators; customs; harbour police; ship owners and/or shipping lines; companies of different transportation modes; insurance companies; banks; customers or cargo receiving companies; and other supply chain stakeholders.

Upon a vessel moors at the quay in the seaport, all cargo-related files are processed by the port agent. Thereby, the necessary information that are usually recorded on physical paper documents (e.g. bill of lading, etc.) are forwarded to the comprehensive INPLAN Port Management System at Wismar seaport. INPLAN eases among other things the forwarding of relevant data and information to the majority of process-involved stakeholders and thus, supports the handling of the respective cargo. Nevertheless, the two main pitfalls are that firstly, the entire operation process flow of the port is not covered and monitored by the system; and secondly, not all internal and especially external stakeholders are directly connected to the system. Consequently, the communication between all involved parties is not optimal enabled and in many cases inefficient through outdated channels leading to the presence of fragmented databases, slowdowns of the process flows and an underperforming supply chain (Prause, 2014).

In the course of a conducted potential analysis of a possible permissioned blockchain implementation, it was derived that the technology fosters the data exchange under the involved parties in the entire process. All relevant cargo-related information can be stored on the ledger of the blockchain once the corresponding participants have approved the uploaded documentation or data, respectively. At the same time the use of the ledger of the blockchain guarantees access to the data and in some cases filtered information at all times and in real time for all participants - including also external stakeholders - if permission is granted. Next to the data reading permission, specific parties can be additionally equipped with a data writing permission. Thereby, data security is ensured through the hashing as well as validation and verification mechanisms that are applied in the course of the creation of blocks to the chain. Next to the hashing and consensus mechanism, all participants receive a copy of the ledger, which jointly secures data integrity in a comprehensive manner and thus, prevent fraud and tampering as well as fosters trust among all involved parties. Overall, the blockchain implementation could streamline in particular the information flow and decreases transaction costs, and thus, the total transportation costs. Furthermore, the implementation of IoT sensors with a direct linkage to the blockchain could provide the distributed and decentralised network with real-time data at all times about the cargo condition and location. For instance, nowadays, especially wood logs are already secured after deforestation by GPS trackers against theft. Thanks to a GPS tracker, which is located in the wood log, it is always possible to trace where it is. Through this, especially the cargo process flow can be optimised due to provided additional cargo-related information that support cargo handling activities and immediately indicate the need for action in case of cargo quality impairment by additional sensor integration (e.g. humidity sensors). Therefore, fewer personnel for quality control checks is required, and error identification in realtime is ensured, which leads to a speed up detection of error causes as well as faster and more efficient countermeasures that can be earlier performed, which heavily saves costs. Accordingly, this is a first step towards automation of the entire port logistics process.

Moreover, by using smart contracts, the automated check of actions and transaction is realised, which leads to a stronger integration of financial service providers into the supply chain. In addition, the payment approval of transactions can be automatically triggered in case of the fulfilment of pre-defined actions. This would improve on a first glance especially the financial flow of the supply chain. However, since all three flows (i.e. information, cargo and financial flow) exhibit interrelations, and thus, are dependent from each other, the comprehensive optimisation of the entire process through the blockchain and smart contract applications forms the real power and synergetic added value of the technology implementation towards a fully automation of port logistics processes. This is also facilitated through the absence of information intermediaries like the port agent or at least lower manual works and responsibilities that are currently covered by the port agent, since all relevant data is directly shared 
among the distributed and decentralised network of stakeholders, which streamline the overall port logistics process flow and reduces the total supply chain costs. The implementation of blockchain and smart contract solutions break down the central control and information system architecture, and by doing so, in the frame of port logistics processes, fosters existing as well as novel entrepreneurial collaborations of different actors in the comprehensive port environment and along the supply chain. This is especially interesting and beneficial for SMEs due to low participation costs after established blockchain platform and connected smart contract applications.

From comparable studies (Prause, 2018), we can assume a reduction of administration costs of about $2 \%$ of total costs induced by the implementation of a blockchain smart contracting system. In addition to that, shorter process times generate additional cost savings. Moreover, as shown in the previous case study, in the course of charter-party contracts for maritime freight transports, smart contracts further encase the potential to replace intermediaries like shipbrokers, which is value adding for all involved supply chain actors (i.e. also for ports) due to spill-over effects. Since within voyage charters the commission of the shipbroker may vary between 1 and $5 \%$ of the gross freight, and in the frame of time charters, the brokerage fee amounts typically $2.5 \%$ of the hire price for the ship; the overall administration cost savings for a seaport like the medium-sized one of Wismar that are attributable to a potential blockchain application including smart contracts amounts at least 4 to $5 \%$. In addition to that, the possible replacement of further information intermediaries like port agents or at least lower manual works and responsibilities that are currently covered by the port agents, less needed personnel for quality control checks, ensured cargo tracking and tracing, as well as earlier countermeasures in case of detected cargo quality impairments in real-time through integrated IoT devices, sensors and trackers in the operative port processes can generate additional direct cost savings. Accordingly, once again it can be derived that the implementation of a blockchain smart contracting system and thus, especially the exclusion of information intermediaries is value adding for all stakeholders of a supply chain. In line with the previous case, also this case study of a cargo import case highlights that in particular for smaller and entrepreneurial actors the implementation of a blockchain smart contracting system fosters competiveness, increases efficiency and facilitate participation through the enabling adoption of this new technology due to arising lower total supply chain costs.

\section{Conclusion}

Smart contracts as transactional scripts on the top of the blockchain technology that both have far reaching potentials to ensure disintermediation in maritime supply chains comprising the capability to reduce transaction and enforcement costs. The research revealed that smart contracts and blockchain technology ease entrepreneurial collaborations of cross-organisational business-processes that are distinguishing for smart supply chains.

Especially, the presented case study on macro-logistics level - here: charter-party contracting process within a single voyager in the break-bulk market - showcased the potentials of the blockchain technology and smart contracts to simplify the business processes in the freight market with increased efficiency. All identified aspects in the course of the optimised process flow decrease costs due to shorten process time and lower manual works. Additionally, the potential exclusion of intermediaries (here: shipbrokers) is in particular value adding for smaller and entrepreneurial actors, since their competiveness and efficiency may be enhanced and participation safeguarded through the enabling adoption of this novel technology due to the emergence of spill-over effects.

Specifically, the case study on micro-logistics level - here: the seaport of Wismar and the related cargo import case - highlighted the potentials of the blockchain technology and smart contracts to simplify the port logistics processes with increased efficiency. Thereby, the benefits of an integration of a blockchain smart contracting system in the ecosystem of port logistics are manifold. For example, by using a blockchain platform for a comprehensive and distributed data and information storing and sharing, and smart contracts for automatically executing specific actions and transaction, as well as with support of IoT devices, sensors and trackers, the entire cargo flow in the port area becomes trackable. This can be regarded as an essential development step towards a partly or fully automated operational process flow, which is crucial for the envisaged objective to become a "smart port". Thereby the IoT devices and sensors deliver the needed information for the smart contract applications, whereby the generated IoT real-time data is stored on the blockchain and thus, visible for all involved network participants. Moreover, especially the incorporation of GPS or other tracking systems and sensors encourage this desired development towards a smart port.

Moreover, based on both case studies, it can be concluded that higher flexibility is given for relevant data sharing, since blockchain represents an open platform that is accessible for everyone who 
shows interest in participation with low entry costs. Through this, the common platform can replace historically grown incompatible systems of different internal and external business entities. Vice versa, this supports a better supply chain integration of all actors.

The common ledger storing procedure ensures real-time and all time distributed network access and information retrieval for all involved parties. Accordingly, no physical documents are needed and each authorised party is able to sign digital with a private key, verify data and fetch copies. Within a permissioned blockchain, it becomes feasible to specify the entree and alteration rights as well as proof of personal identification. Moreover, in a permissioned blockchain, some information can stay private or can be shared in a filtered form, which prevent a potential data flooding.

As a side effect simultaneous file work and a transparent process tracking is always ensured. Generally, through blockchain implementation, all information are decentralised and distributed secured, fraud-resistant, irreversible, steady verifiable, retraceable and always retrievable. All these features increase trust under the authorised network participants and enhance efficiency and flexibility of all process flows (cargo, financial, information).

Smart contracts are able to automatically create and distribute important documents (e.g. charterparty contract, bill of lading, etc.). Therefore, also the auditing process of documents and relevant external data that is provided by IoT sensors and trackers is automated by smart contract applications. Through the programmed processing of this data, final calculations can be automated, too. This automated billing represents a fair and trustful procedure of expenditure calculation and financial transactions.

All these identified advantages that arise from the introduction of a blockchain and smart contracting system decrease costs, which can be traced back to streamlined process time and less manual actions (e.g. cargo quality checks, information auditing procedures, physical paper and document forwarding via courier, etc.). In addition, especially the reduction or elimination of information intermediaries is beneficial for small and entrepreneurial actors, which fosters their competiveness and process efficiency. This is, because they face lower transaction costs and low entry barriers induced by disintermediation. This illustrates that smart contracts and blockchain in the areas of maritime supply chains support the integration of entrepreneurs and SMEs into trans-national maritime value and supply chains by reducing high entry barriers and by weakening the dominating position of big players.

Future research activities should have a deeper look on the legal aspects that must be overcome by introducing blockchains and smart contracting systems in maritime supply chains and in port logistics, as so far they have not been discussed in this research study. At the same time, this represents a general limitation of the current research. Moreover, since the research on smart contracts and blockchain technology in the nexus of maritime logistics sector, and in particular in the context of small and mediumsized seaports have not sufficiently investigated so far, a methodological limitation of the present research study is obvious due to lack of prior research studies on the topic. On the other hand, this represented a clear research gap that needed to be closed. Therefore, the addressed research field of the current research study expresses a high novelty value and originality, since the focus is dedicated to the challenging and upcoming digitalisation issues that arise in case of maritime supply chains and particularly in the context of small and medium-sized ports.

\section{Acknowledgements}

This research article has been supported by the European Regional Development Fund (INTERREG VA South Baltic and INTERREG VB Baltic Sea Region programmes) within the projects "Connect2SmallPorts" and "CSHIPP".

\section{References}

1. Beth, S., Burt, D. N., Copacino, W., Gopal, C., Lee, H. L., Lynch, R. P., \& Morris, S. (2003). Supply chain challenges. building relationships. Harvard business review, 81(7), 64-73.

2. Caliskan, A., \& Ozturkoglu, Y. (2018). Maritime logistics. In Intelligent Transportation and Planning: Breakthroughs in Research and Practice (pp. 822-845). IGI Global.

3. Chuen, D. L. K. (Ed.). (2015). Handbook of digital currency: Bitcoin, innovation, financial instruments, and big data. Academic Press.

4. Creswell, J. W., \& Creswell, J. D. (2017). Research design: Qualitative, quantitative, and mixed methods approaches. Sage publications.

5. European Commission (2017). Report on the Blue Growth Strategy - Towards more sustainable growth and jobs in the blue economy. Commission staff working document. SWD(2017) 128 final, Brussels, 31.3.2017. 
6. Eurostat.(2019a) http://appsso.eurostat.ec.europa.eu/nui/show.do?dataset=mar_mg_aa_cwh\&lang=en (accessed: July 2019)

7. Eurostat. (2019b) http://appsso.eurostat.ec.europa.eu/nui/show.do?dataset=mar_mp_aa_cph\&lang=en (accessed: July 2019).

8. Funke, M., \& Yu, H. (2011). The emergence and spatial distribution of Chinese seaport cities. China Economic Review, 22(2), 196-209.

9. Gallay, O., Korpela, K., Tapio, N., \& Nurminen, J. K. (2017). A peer-to-peer platform for decentralized logistics. In: Proceedings of the Hamburg International Conference of Logistics (HICL), pp. 19-34. epubli.

10. García-Bañuelos, L., Ponomarev, A., Dumas, M., \& Weber, I. (2017). Optimized execution of business processes on blockchain. In: International Conference on Business Process Management, pp. 130-146. Springer, Cham.

11. Gerlitz, L. (2015). Design for product and service innovation in Industry 4.0 and emerging smart society. Journal of Security \& Sustainability Issues, 5(2).

12. Gerlitz, L. (2017). Design-driven innovation in SMEs: smart and sustainable organisation within industrial transformation. International Journal of Environmental Policy and Decision Making, 2(2), 98-124.

13. Kouhizadeh, M., \& Sarkis, J. (2018). Blockchain Practices, Potentials, and Perspectives in Greening Supply Chains. Sustainability, 10(10), 3652.

14. L'Hermitte, C., Wang, W., \& Deakins, E. (2018). Exploring the Physical Internet Concept to Improve Disaster Relief Operations.

15. Liao, D. Y., \& Wang, X. (2018). Applications of Blockchain Technology to Logistics Management in Integrated Casinos and Entertainment. Informatics, 5(4), 44. Multidisciplinary Digital Publishing Institute.

16. Manski, S. (2016). Building the blockchain: the co-construction of a global commonwealth to move beyond the crises of global capitalism. In: 12th Annual California Graduate Student Conference, 7th May.

17. Nakamoto, S. (2008). Bitcoin: A peer-to-peer electronic cash system, http://bitcoin.org/bitcoin.pdf (accessed: July 2019)

18. Nyhuis, P., \& Wiendahl, H. P. (2008). Fundamentals of production logistics: theory, tools and applications. Springer Science \& Business Media.

19. Olaniyi, E. O., \& Reidolf, M. (2015). Organisational Innovation Strategies in the Context of Smart Specialisation. Journal of Security \& Sustainability Issues, 5(2).

20. Pastori, E. (2015). Modal share of freight transport to and from EU ports. Policy Department B: Structural and Cohesion Policies European Parliament: Brussels, Belgium.

21. Philipp, R., Gerlitz, L. \& Prause, G. (2018). Regionale Häfen auf Digitalisierungskurs: Intelligentes Wachstum und nachhaltige Wertschöpfung entlang der kleinen und mittel-großen Häfen des Ostseeraumes, In: Cleve, Alde, Wißotzki (eds.): Proceedings of WiWiTa 2018 Conference, 77 - 86, Wismar, ISBN 978-3-942100-58-8, https://www.researchgate.net/publication/326113557 (accessed: July 2019)

22. Prause, G. (2014). A Green Corridor Balanced Scorecard. Transport and Telecommunication, 15 (4), 299-307. DOI:10.2478/ttj-2014-0026.

23. Prause, G. (2015). Sustainable business models and structures for Industry 4.0. Journal of Security \& Sustainability Issues, 5(2), 159-169. DOI:10.9770/jssi.2015.5.2(3).

24. Prause, G. (2018). e-Government als Standortfaktor, In: Cleve, Alde, Wißotzki (eds.): Proceedings of WiWiTa 2018 Conference, 14-20, Wismar, ISBN 978-3-942100-58-8.

25. Prause, G. (2019). Smart Contracts for Smart Supply Chains. In: 9th IFAC/IFIP/IFORS/IISE/INFORMS Conference Manufacturing Modelling, Management and Control (MIM 2019), Berlin.

26. Prause, G., \& Atari, S. (2017). On sustainable production networks for Industry 4.0. Entrepreneurship and Sustainability Issues, 4(4), 421-431.

27. Prause, G., \& Hoffmann, T. (2017). Cooperative Business Structures for Green Transport Corridors. Baltic Journal of European Studies, 7(2), 3-27.

28. Prause, G., \& Hunke, K. (2014). Secure and Sustainable Supply Chain Management: Integrated ICTSystems for Green Transport Corridors. Journal of Security \& Sustainability Issues, 3(4), 5-16. DOI:10.9770/jssi.2014.3.4(1).

29. Rodrigue, J. P., \& Schulman, J. (2013). The Economic Impacts of Port Investments. The Geography of Transport Systems. 
30. Rozmarynowska, M., \& Oldakowski, B. (2013). Development perspectives for small and medium Baltic Sea ports. In: The TransBaltic and Baltic Ports Organization's Seminar.

31. Rusich, A. (2017). Collaborative Logistics Networks. Diss., University of Trieste, CIRRELT-201750, Trieste (August 2017).

32. Simchi-Levi, D., Kaminsky, P., \& Simchi-Levi, E. (2003). Design and managing the supply chain, McGraw Hill Professional, ISBN 9780072492569, 354 p.

33. Stadtler, H. (2005). Supply chain management and advanced planning-basics, overview and challenges. European journal of operational research, 163(3), 575-588.

34. Stake, R. E. (1995). The art of case study research. Sage.

35. Swan, M. (2015). Blockchain: Blueprint for a new economy. "O'Reilly Media, Inc.".

36. Wehberg, G. G. (2016). Logistik 4.0: Komplexität managen in Theorie und Praxis. Springer Gabler.

37. Wu, L. (2018). Blockchain Smart Contracts in Megacity Logistics. The Pennsylvania State University.

38. Yin, R. (2009). Case Study Research: Design and Methods, SAGE Publications. California, USA.

39. Zhao, L., \& Huchzermeier, A. (2018). Supply chain finance. In: Supply Chain Finance, 105-119. Springer, Cham. 